\title{
BMJ Open Choice of anaesthetic in emergency operations: a protocol for a scoping review
}

\author{
Lucy Elliott (D) , ${ }^{1,2}$ Rachael L Morley (D) ,, ${ }^{1,2}$ Ronelle Mouton, ${ }^{1,2}$ \\ Robert Hinchliffe (D) ${ }^{1,3}$
}

To cite: Elliott L, Morley RL, Mouton R, et al. Choice of anaesthetic in emergency operations: a protocol for a scoping review. BMJ Open 2019;9:e030817. doi:10.1136/ bmjopen-2019-030817

- Prepublication history and additional material for this paper are available online. To view these files, please visit the journal online (http://dx.doi. org/10.1136/bmjopen-2019030817).

LE and RLM are joint first authors.

Received 04 April 2019

Revised 22 September 2019

Accepted 03 October 2019

\begin{abstract}
Introduction The effect of mode of anaesthesia in emergency surgery is uncertain. This scoping review will identify and summarise the evidence for local, regional or general anaesthetic in adult patients undergoing emergency surgical procedures.
\end{abstract}

Methods and analysis Scoping review methodology will be followed. The search will be run in EMBASE and Medline. Further articles will be identified from searching references in relevant studies. A descriptive analysis will be performed considering the following main outcomes; mortality, length of stay, intensive care unit (ICU) admission, postoperative pain and morbidity. Data on complications including acute coronary syndrome, stroke, thromboembolic events, delirium, acute kidney injury, respiratory tract infection, surgical site infection and studyspecific additional outcomes will also be collected.

Ethics and dissemination No ethics approval is required. The results will be used to inform a funding application for a clinical trial comparing local/regional anaesthetic with general anaesthetic. The study will be disseminated via peer-reviewed manuscript and conferences.

Trial registration number Results will be reported according to the Preferred Reporting Items for Systematic Reviews and Meta-Analyses extension for Scoping Reviews (PRISMA-ScR) Statement. There are currently no registries that accept scoping reviews.

\section{INTRODUCTION}

\section{Rationale}

Elective surgery accounts for the vast majority of research effort and is now generally considered a safe intervention. ${ }^{1}$ Patients undergoing emergency surgery have a significantly higher risk of morbidity and mortality compared with their elective counterparts. ${ }^{2}{ }^{3}$ Despite this, the evidence to support decision-making in emergency surgery is lacking. ${ }^{1}$ Wellconducted research of interventions in emergency surgery have the potential to yield large improvements in these inferior outcomes. Mode of anaesthesia is a possible candidate for this, as currently little is known about their effects on outcome of patients requiring emergency surgery.

\section{Strength and limitations of this study}

This scoping review will summarise the evidence for local/regional anaesthetic compared to general anaesthetic in emergency surgery. No such study has previously been conducted.

- This protocol outlines previously validated scoping review methodology to provide a comprehensive yet time efficient conclusion.

- There will be difficulty in identifying all emergency surgical procedures as not all will be referred to as 'emergency' or 'urgent'. However, the search strategy is comprehensive to cover possible emergency presentations by name.

- Search strategy will be limited by outcomes that are likely to be common to all emergency procedures, These were chosen in the absence of an agreed core outcome set for trials in anaesthetic, being commonly reported in surgical and anaesthetic trials.

Recent analysis of the improve trial revealed a significantly improved mortality outcome in ruptured abdominal aortic aneurysm (AAA) under local rather than general anaesthetic during endovascular AAA repair. ${ }^{4}$ Similar work conducted in stroke thrombectomy has suggested potential benefits for patients when using locoregional anaesthesia instead of general anaesthesia. ${ }^{56}$ This suggests that there are other areas of emergency surgery where mode of anaesthesia is an area of research interest. A scoping review is the most efficient method to identify studies that have already been conducted for emergency surgical procedures and may also provide some insight into outcomes.

\section{Overall objectives}

The aims of this review are as follows:

1. Map existing evidence from studies that compare locoregional with general anaesthesia techniques in adult patients undergoing emergency surgery and to identify potential gaps in knowledge. 
2. Determine whether full systematic review of this literature is possible.

3. Identify needs (if any) for improved reporting within anaesthetic trials in emergency surgery.

\section{METHODS}

\section{Eligibility criteria}

Studies published between 2003 and 2019, comparing use of locoregional and general anaesthetics in surgery of any specialty in human adults over 18 years of age, will be included. Only primary research studies will be included.

For this review, 'emergency' will be defined according to the National Confidential Enquiry into Patient Outcome and Death classification of 'immediate' or 'urgent' procedure. ${ }^{7}$ It is envisaged that papers will identify procedures as emergency or urgent. However, if not otherwise stated, any description that would indicate an emergency repair will be included, for example, strangulated, non-reducible, obstructed. If not otherwise definable by the above, emergency procedures will be defined as unplanned admissions where it is not possible to discharge the patient home before their operation. ${ }^{1}$

For this review, the types of anaesthesia to be studied will be conscious sedation, local, subarachnoid, epidural, regional nerve and general anaesthetic. Papers in which multimodal anaesthesia is studied will be excluded, unless single modes are also compared.

Only full text studies in English will be included but there will be no limit on country of study. Reports on anaesthetic in elective operations will not be included unless they also report a subgroup of emergency cases that can be analysed separately. All comparative studies will be included, including non-randomised and retrospective reports. Case reports and other non-comparative studies will be excluded.

The following clinical postoperative outcomes will be included; mortality, hospital length of stay, intensive care unit (ICU) admission, pain score, acute coronary event, stroke, thromboembolic event, delirium, lower respiratory tract infection, surgical site infection, acute kidney injury, overall morbidity and any additional study-specific outcomes.

\section{Information sources}

The search will be run in EMBASE and Medline. Further articles will be identified from searching references in relevant studies. In addition, the senior authors ( $\mathrm{RJH}+$ RM) identified studies from their own respective research fields so that studies including relevant post hoc analysis not identified in the initial search were also included in our review. No grey literature will be searched as this scoping review is intended to identify papers that may be available for a full systematic review. A detailed summary of the search including MeSH terms is included in online supplementary appendix 1 .

\section{Search strategy}

The search has been developed with the help of an experienced academic librarian. Search results will be limited to 2003-2019 and to articles written in English. There will be no limit on country of study. The search will include general terms to identify emergency procedures (eg, emergency/urgent) as well as more specific words that may be used (eg, ruptured, incarcerated, torsion).

\section{Study records}

This scoping review will be conducted using the methodological framework described by Arksey and O'Malley and reported according to the Preferred Reporting Items for Systematic Review and Meta-analysis Protocols Extension for Scoping Reviews (PRISMA-ScR) checklist. ${ }^{89}$

\section{Data management}

Screening and data collection will be done by one reviewer on Excel (Microsoft, 2018). The proposed data collection form is detailed in online supplemntary appendix 2.

\section{Selection process}

Two authors will independently review abstracts and full texts according to the preagreed inclusion criteria.

\section{Data collection process}

Data will be extracted by one reviewer and verified by a second reviewer. The data collection form will be piloted with four studies and any issues resolved between authors before further data collection.

\section{Data items}

Data will be sought for the following demographic variables:

Patient gender as male to female ratio, average patient age in years including range, surgical specialty and surgical operation, American Society of Anesthesiologist grade if available, country of study, description of anaesthesia as an intervention as reported in the study, reference to anaesthetic protocol in study, study design (retrospective or prospective) and study type (cohort, case-control or randomised control trial).

\section{Data synthesis}

The review will be reported in accordance with Preferred Reporting Items for Systematic Review and Meta-Analysis reporting guidelines for scoping reviews.

Results will be presented in tabulated form and as a narrative synthesis of outcomes by mode of anaesthesia. There will be no quantitative data analysis as data collected is will span a number of procedures and is expected to be very heterogenous. Outcomes will be discussed individually in a narrative synthesis.

\section{Consultation phase}

An integrated consultative approach will be adopted, whereby senior members of the scoping team, including experts in their respective fields of surgery (RJH) and anaesthesia (RM), are informed of the results of the 
literature review and the data extracted from included papers and through an iterative process, will guide additional data extraction from the papers as deemed necessary. This is in keeping with established scoping review methodology. ${ }^{10}$

\section{PATIENT AND PUBLIC INVOLVEMENT}

There was no involvement in patients or public in the design of this study, and the research question was not formulated or developed with patient or public involvement. Additionally, no patients or public members are required in order to complete this scoping review.

\section{ETHICS AND DISSEMINATION}

No ethical approval is required for scoping reviews. The study will be broadly disseminated through via peerreviewed manuscript published in an open-access journal and presentation at conferences.

The results will be used to inform a funding application and design for a clinical trial comparing local/ regional anaesthetic with general anaesthetic in emergency surgical procedures. This will aid to improve outcomes in a population with a typically poor outcome and minimal evidence base. ${ }^{1}$ No such work has previously been undertaken.

Contributors I can confirm that all authors named have contributed fully to the concept and design of the scoping review for which this protocol has been written. RLM contributed to conception and design of the study and has written and reviewed the manuscript before submission. LE contributed to conception and design of the study, has written and reviewed the manuscript before submission. RLM + LE are joint first authors. RM contributed to conception and design of the study and has reviewed the manuscript before submission. RH contributed to conception and design of the study and has reviewed the manuscript before submission.

Funding This work was undertaken with the support of the David Telling grant at North Bristol NHS Trust. It was supported by the NIHR Biomedical Research Centre at the University Hospitals Bristol NHS Foundation Trust and the University of Bristol.

Competing interests None declared.
Patient consent for publication Not required.

Provenance and peer review Not commissioned; externally peer reviewed.

Open access This is an open access article distributed in accordance with the Creative Commons Attribution Non Commercial (CC BY-NC 4.0) license, which permits others to distribute, remix, adapt, build upon this work non-commercially, and license their derivative works on different terms, provided the original work is properly cited, appropriate credit is given, any changes made indicated, and the use is non-commercial. See: http://creativecommons.org/licenses/by-nc/4.0/.

ORCID iDs

Lucy Elliott http://orcid.org/0000-0002-4265-4075

Rachael L Morley http://orcid.org/0000-0001-7918-4196

Robert Hinchliffe http://orcid.org/0000-0002-6370-0800

\section{REFERENCES}

1 Morley RL, Edmondson MJ, Rowlands C, et al. Registration and publication of emergency and elective randomised controlled trials in surgery: a cohort study from trial registries. BMJ Open 2018;8:e021700.

2 Mullen MG, Michaels AD, Mehaffey JH, et al. Risk associated with complications and mortality after urgent surgery vs elective and emergency surgery. JAMA Surg 2017;152:768.

3 Visser A, Geboers B, Gouma DJ, et al. Predictors of surgical complications: A systematic review. Surgery 2015;158:58-65.

4 IMPROVE Trial Investigators. Comparative clinical effectiveness and cost effectiveness of endovascular strategy $V$ open repair for ruptured abdominal aortic aneurysm: three year results of the improve randomised trial. BMJ 2017;359.

5 Brinjikji W, Pasternak J, Murad MH, et al. Anesthesia-Related outcomes for endovascular stroke revascularization. Stroke 2017;48:2784-91.

6 Campbell BCV, van Zwam WH, Goyal M, et al. Effect of general anaesthesia on functional outcome in patients with anterior circulation ischaemic stroke having endovascular thrombectomy versus standard care: a meta-analysis of individual patient data. Lancet Neurol 2018;17:47-53.

7 National Confidential Enquiry into Patient Outcome and Death. The NCEPOD classification of intervention, 2018. Available: https://www. ncepod.org.uk/classification.html [Accessed 20 Aug 2019].

8 Arksey H, O'Malley L. Scoping studies: towards a methodological framework. Int J Soc Res Methodol 2005;8:19-32.

9 Tricco AC, Lillie E, Zarin W, et al. PRISMA extension for scoping reviews (PRISMA-ScR): checklist and explanation. Ann Intern Med 2018;169:467.

10 O'Brien KK, Colquhoun H, Levac D, et al. Advancing scoping study methodology: a web-based survey and consultation of perceptions on terminology, definition and methodological steps. BMC Health Serv Res 2016;16:305. 